\title{
A common PRRT2 mutation in familial paroxysmal kinesigenic dyskinesia in Hong Kong: a case series of 16 patients
}

\author{
CY Law ${ }^{1 \#, ~ P h D, ~ F H K A M ~(P a t h o l o g y), ~ W L ~ Y e u n g ~}{ }^{2 \#}$, FHKAM (Paediatrics), YF Cheung ${ }^{3 \#, ~ M P H, ~ F R C P, ~}$ \\ HF Chan ${ }^{3}$, FHKAM (Medicine), Eva Fung ${ }^{4}$, FHKAM (Paediatrics), Joannie Hui ${ }^{4}$, FHKAM (Paediatrics), Iris OK Yung ${ }^{5}$, MD, \\ YP Yuen ${ }^{6}$, MSc, FHKAM (Pathology), Angel OK Chan ${ }^{7}$, MD, FHKAM (Pathology), CW Lam ${ }^{1}$, PhD, FHKAM (Pathology) \\ \# These authors contributed equally to this work. \\ ${ }^{1}$ Department of Pathology, The University of Hong Kong, Pokfulam, Hong Kong \\ ${ }^{2}$ Department of Paediatrics and Adolescent Medicine, Alice Ho Miu Ling Nethersole Hospital, Tai Po, Hong Kong \\ ${ }^{3}$ Division of Neurology, Department of Medicine, Queen Elizabeth Hospital, Jordan, Hong Kong \\ ${ }^{4}$ Department of Paediatrics, Prince of Wales Hospital, Shatin, Hong Kong \\ ${ }^{5}$ Division of Neurology, Department of Medicine and Therapeutics, Prince of Wales Hospital, Shatin, Hong Kong \\ ${ }^{6}$ Department of Chemical Pathology, Prince of Wales Hospital, Shatin, Hong Kong \\ ${ }^{7}$ Division of Clinical Biochemistry, Queen Mary Hospital, Pokfulam, Hong Kong \\ *Corresponding author: ching-wanlam@pathology.hku.hk
}

Hong Kong Med J 2016;22:619-22

DOI: 10.12809/hkmj154579

\section{Case reports}

\section{Family 1}

Patient 1 was a 47-year-old woman who presented with paroxysmal abnormal movement since childhood. She was first seen in 2004. The attack was characterised by dystonic painful posture with upward or downward deviation of the eyes, head turning, shoulder abduction and extensions, lower limb dystonia, impaired speech, and occasionally stepping movements, with preserved consciousness. The attacks lasted for 1 to 2 minutes with a frequency of up to 8 times a day. Each attack was precipitated by stress or being startled, for example, being approached by a car unexpectedly when crossing the road, frightened by a cockroach, a sudden phone call, or an abrupt movement. Occasionally, the attack could occur during sleep. There was no incontinence. The patient had normal intelligence. Physical examination, biochemical tests, computed tomography of the brain, and electroencephalography were normal. The attacks were controlled with carbamazepine and frequency of attacks reduced to fewer than 3 times a day and each episode was shortened to 10 to 20 seconds. The patient also had a history of seizure of unknown aetiology in childhood. She had a strong family history; her daughter has presented with seizure on two to three occasions since the age of 10 months and had been taking an anticonvulsant for 2 years. Since then she had experienced no further seizures and by the age of 4 years no antiepileptic drug or follow-up has been required. Two older sisters and a nephew of the proband also had childhood seizures.
Family 2

Patient 2 was a 26-year-old woman who presented with paroxysmal kinesigenic dyskinesia (PKD) since the age of 8 years. The patient first presented with episodes of afebrile seizure at 7 months old in 1997. She was prescribed phenobarbitone and required no further medication or follow-up after the age of 3 years. The current involuntary movement affected all limbs with the right side being most affected. Each attack lasted for a few seconds to less than 3 minutes without loss of consciousness. Each episode was triggered by sudden body movement, anxiety, or deprived sleep. Frequency of attacks could reach up to 6 times a day. The patient claimed she could occasionally partially control the symptoms. Physical examination, biochemical tests, and brain imaging were unremarkable. Her brother was also affected by PKD. The attack was controlled by carbamazepine; a missed dose would usually lead to relapse.

\section{Family 3}

Patient 3 was a 14-year-old boy who presented in 2004 with chorea-like movement of the four limbs since the age of 18 months. Each attack lasted for about 15 to 20 seconds and there was no impaired consciousness. The frequency of attacks could reach up to 10 times a day. The attacks were triggered after running or waking up from sleep. A good clinical response was achieved with carbamazepine. The attacks recurred only after a missed dose. Physical examination, biochemical investigations, and brain imaging were unremarkable and he had normal development and intelligence. Five paternal family 
members were similarly affected.

\section{Family 4}

Patient 4 was a 33-year-old man who presented with involuntary movement since the age of 9 years. He was first seen in 2011. The involuntary body movement affected his right facial muscle and right upper and lower limbs. The attack usually lasted for 5 to 10 seconds with no impaired consciousness. No remarkable triggering factor was noted. Physical examination, biochemical tests, and brain imaging were unremarkable. The patient also experienced migraine triggered by increased stress. He had been prescribed carbamazepine since the age of 10 years and a missed dose would lead to relapse. Two of his daughters were affected by seizures. The elder daughter presented at 7 months old with two episodes of generalised seizure, each lasting around 30 seconds. The younger daughter presented at 3 months old with a brief lip smacking, limb twitching, and up-rolling eyeball; the attack lasted about 2 minutes. Both daughters were prescribed phenobarbitone. They achieved normal growth and no further antiepileptic drug was required at 34 months of age for the elder sister while the younger sister continued on low-dose phenobarbitone.

\section{Family 5}

Patient 5 was a 14-year-old boy who presented with PKD since the age of 9 years. He presented with on-and-off dystonic movement involving the limbs, trunk, and occasionally the face. The attacks lasted for less than 10 seconds and there was no impaired consciousness. Each episode was triggered by sudden body movement, for example, starting to write or going out of an elevator. The frequency of attacks ranged from once every 2 days to twice a day. Physical examination and biochemical investigations were unremarkable. Magnetic resonance imaging of the brain showed an incidental finding of left temporal arachnoid cyst. A trial of carbamazepine achieved a good clinical response.

\section{Family 6}

Patient 6 was a 29-year-old man who presented with PKD around the age of 3 years in 1988. He presented with jerky variable involuntary movement of all limbs without loss of consciousness. The attacks usually lasted for less than 1 minute and were triggered by sudden body movements. The attacks were not precipitated by alcohol, caffeine, fatigue, stress, or excitement. The frequency of attacks could be daily. Aura was noted before dystonic movement. Physical examination, biochemical tests, and brain imaging were unremarkable. The attacks were effectively controlled by carbamazepine. His father had been similarly affected since his teenage years. Each attack usually lasted for 1 to 2 minutes, precipitated by prolonged sitting or excitement. Aura was noted before dystonic movement. The sister of the proband was mildly affected with sudden tightness and stiffness of limbs without impaired consciousness. Aura was reported before the dystonic movement. The other three siblings of the proband were asymptomatic.

\section{Mutational analysis for PRRT2 gene}

Exons of PRRT2 were amplified using polymerase chain reaction, of which its conditions and primer sequences for PRRT2 are available upon request. Sequencing results were compared with the National Center for Biotechnology Information reference sequences NM_145239.2 and NP_660282.2. Mutation analysis for PRRT2 gene in families 1 to 5 showed a heterozygous frameshift mutation c.649dupC (p.Arg217Profs*8) [Fig 1]. This is a known pathogenic mutation that can result in premature transcription termination and a truncated PRRT2 protein. For family 1, DNA samples were not available for other affected family members except

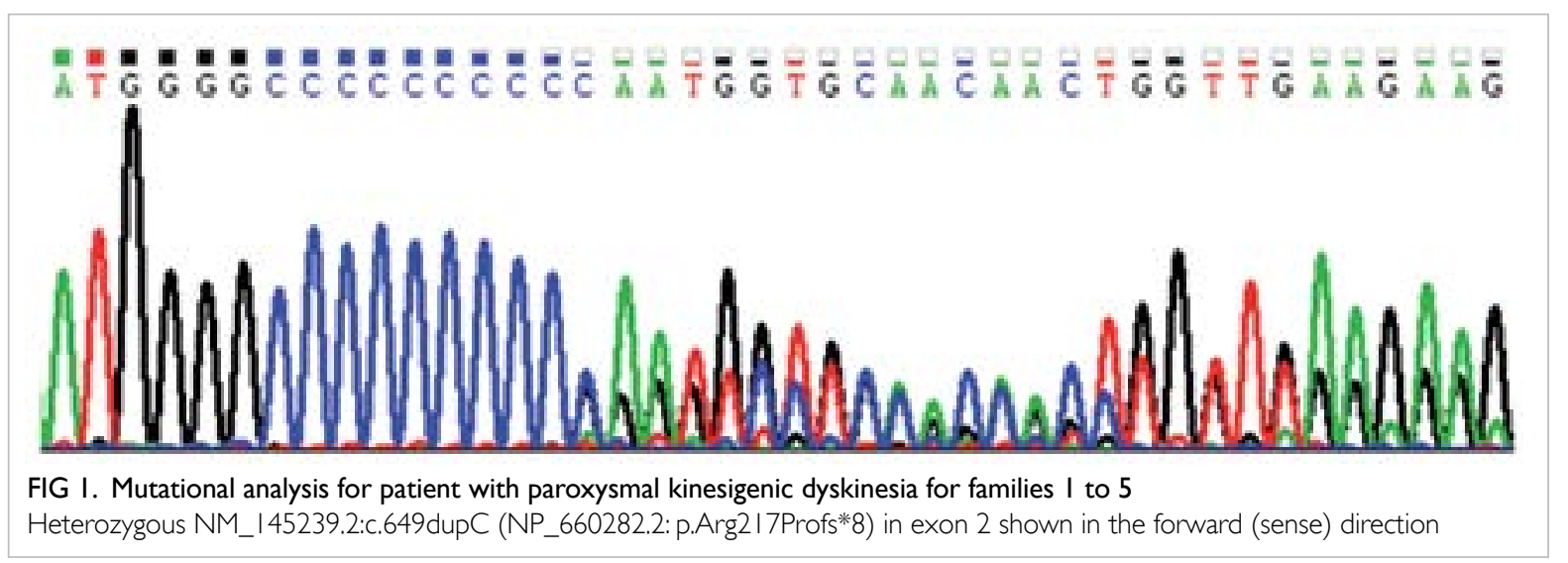




\section{C}

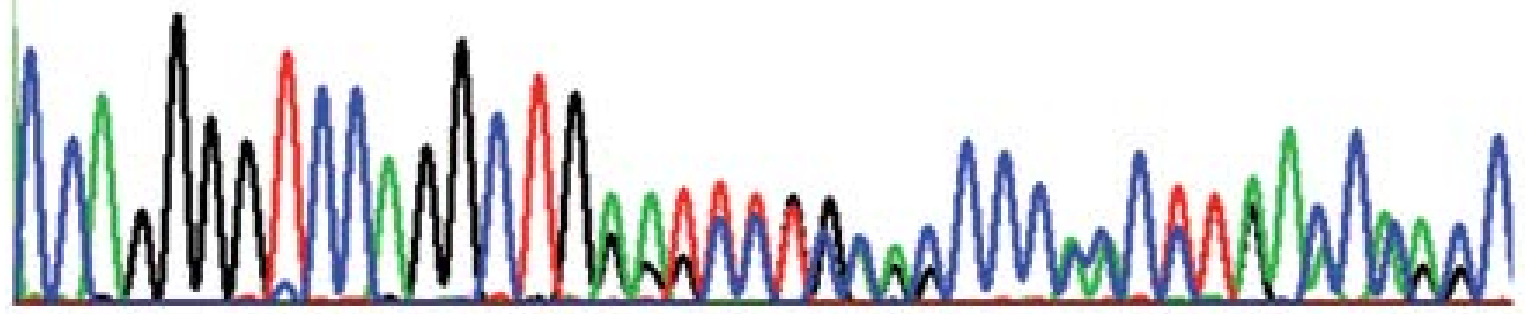

FIG 2. Mutational analysis for patient with paroxysmal kinesigenic dyskinesia for family 6

Heterozygous NM_I 45239.2:c.379delG (NP_660282.2: p.Glu I27Serfs*49) in exon 2 shown in the forward (sense) direction

the proband. For family 6, a novel heterozygous frameshift mutation, c.379delG (p.Glu127Serfs*49), was detected (Fig 2). The mutation will lead to premature transcription termination and a truncated PRRT2 protein.

\section{Discussion}

Familial PKD (OMIM\#128200) is the most common type of paroxysmal movement disorder. It is defined by the presence of (1) identified kinesigenic triggers, (2) short duration of attacks, usually $<1$ minute, (3) no loss of consciousness or pain during attacks, (4) absence of known organic causes and normal neurological examination, (5) response to phenytoin or carbamazepine treatment, and (6) age of onset at 1 to 20 years in the absence of a family history of PKD. ${ }^{1}$ The attack is characterised by the presence of involuntary movement such as dystonia, choreoathetosis, and ballism. The co-existence of PKD and infantile convulsions can occur in a subset of PKD patients and is known as PKD with infantile convulsions (PKD/IC).

The disorder PKD is transmitted as an autosomal dominant trait with incomplete penetrance. The disease is caused by mutation of the PRRT2 gene (proline-rich transmembrane protein 2; OMIM*614386). ${ }^{2,3}$ PRRT2 mutation can also cause $\mathrm{PKD} / \mathrm{IC}$, paroxysmal exercise-induced dyskinesia, paroxysmal non-kinesigenic dyskinesia, benign familial infantile epilepsy, episodic ataxia, hemiplegic migraine, intellectual disability, and benign paroxysmal torticollis of infancy. ${ }^{4,5}$ Patients with PKD can be misdiagnosed with epilepsy or psychogenic illness ${ }^{6}$; an aetiological diagnosis will require genetic analysis of $P R R T 2$ gene, the most common disease-causing gene for PKD.

PRRT2 mutation is the major cause of PKD in Chinese, Koreans, Japanese, and Europeans ${ }^{7-11}$ and accounts for $33 \%$ to $46 \%$ of sporadic and $80 \%$ to $100 \%$ of familial forms of PKD. ${ }^{12}$ Heterozygous c.649dupC is a mutation hotspot of which the mutation detection rate for Chinese can be as high as $62 \% .{ }^{13}$ Our findings also suggest that the majority (81.3\%; 95\% confidence interval, 57.0\%-93.4\%) of PKD patients (13 out of 16) carried the c.649dupC mutation.

Most (79\%) PKD patients had a distinctive phenotype. ${ }^{1}$ Atypical features have been reported, for example, long duration of attack, ${ }^{2}$ attacks triggered by stress/anxiety, ${ }^{3}$ and painful dystonia. ${ }^{14}$ This explains the unusual features observed in family 1 (painful dystonia, attacks precipitated by stress) and family 2 (attacks up to 3 minutes). Intriguingly, no obvious triggering factors were identified in family 4 , likely because they were too subtle and not recognised by the patient.

Co-existence of infantile convulsion can occur in a subset of PKD patients (ie PKD/IC), as with the proband in families 1 and 2. Nevertheless, PKD was not apparent in the affected children of families 1 and 4, likely because the mean age of onset was $11.6 \pm 3.5$ years, ${ }^{1}$ and they were too young for PKD manifestation.

An aetiological diagnosis for patients with PKD is clinically important. First, the majority of patients show an excellent response to carbamazepine or phenytoin and some show significant improvement with low-dose carbamazepine. ${ }^{15}$ Second, neurologists, paediatricians, and pathologists can explain to the family the aetiology of PKD. Third, patients can be misdiagnosed with epilepsy or 'psychogenic illness'. A definitive diagnosis can end the diagnostic uncertainty and relieve patients of the emotional uncertainty and unnecessary investigations.

In conclusion, the cases reported here constitute the first genetic-confirmed series of PKD in Hong Kong. We recommend PRRT2 c.649dupC screening for all patients with all forms of PKD. 


\section{References}

1. Bruno MK, Hallett M, Gwinn-Hardy K, et al. Clinical evaluation of idiopathic paroxysmal kinesigenic dyskinesia: new diagnostic criteria. Neurology 2004:63:2280-7.

2. Chen WJ, Lin Y, XiongZQ, et al. Exome sequencing identifies truncating mutations in PRRT2 that cause paroxysmal kinesigenic dyskinesia. Nat Genet 2011;43:1252-5.

3. Wang JL, Cao L, Li XH, et al. Identification of PRRT2 as the causative gene of paroxysmal kinesigenic dyskinesias. Brain 2011;134:3493-501.

4. Liu Q, Qi Z, Wan XH, et al. Mutations in PRRT2 result in paroxysmal dyskinesias with marked variability in clinical expression. J Med Genet 2012;49:79-82.

5. Gardiner AR, Bhatia KP, Stamelou M, et al. PRRT2 gene mutations: from paroxysmal dyskinesia to episodic ataxia and hemiplegic migraine. Neurology 2012;79:2115-21.

6. Matsumoto N, Takahashi S, Okayama A, Araki A, Azuma $\mathrm{H}$. Benign infantile convulsion as a diagnostic clue of paroxysmal kinesigenic dyskinesia: a case series. J Med Case Rep 2014;8:174.

7. Chen YP, Song W, Yang J, et al. PRRT2 mutation screening in patients with paroxysmal kinesigenic dyskinesia from Southwest China. Eur J Neurol 2014;21:174-6.

8. Youn J, Kim JS, Lee M, et al. Clinical manifestations in paroxysmal kinesigenic dyskinesia patients with prolinerich transmembrane protein 2 gene mutation. J Clin
Neurol 2014;10:50-4.

9. Okumura A, Shimojima K, Kubota T, et al. PRRT2 mutation in Japanese children with benign infantile epilepsy. Brain Dev 2013;35:641-6.

10. Méneret A, Grabli D, Depienne C, et al. PRRT2 mutations: a major cause of paroxysmal kinesigenic dyskinesia in the European population. Neurology 2012;79:170-4.

11. Becker F, Schubert J, Striano P, et al. PRRT2-related disorders: further PKD and ICCA cases and review of the literature. J Neurol 2013;260:1234-44.

12. Labate A, Tarantino $\mathrm{P}$, Viri $\mathrm{M}$, et al. Homozygous c.649dupC mutation in PRRT2 worsens the BFIS/PKD phenotype with mental retardation, episodic ataxia, and absences. Epilepsia 2012;53:e196-9.

13. Cao L, Huang XJ, Zheng L, Xiao Q, Wang XJ, Chen SD. Identification of a novel PRRT2 mutation in patients with paroxysmal kinesigenic dyskinesias and c.649dupC as a mutation hot-spot. Parkinsonism Relat Disord 2012;18:704-6.

14. Ebrahimi-Fakhari D, Kang KS, Kotzaeridou U, Kohlhase J, Klein C, Assmann BE. Child Neurology: PRRT2-associated movement disorders and differential diagnoses. Neurology 2014;83:1680-3.

15. Chou IC, Lin SS, Lin WD, et al. Successful control with carbamazepine of family with paroxysmal kinesigenic dyskinesia of PRRT2 mutation. Biomedicine (Taipei) 2014;4:15 